\title{
Numerical Study on Stability of Rock Slope Based on Energy Method
}

\author{
Wei Gao, Xu Wang, Shuang Dai, and Dongliang Chen \\ Key Laboratory of Ministry of Education for Geomechanics and Embankment Engineering, College of Civil and \\ Transportation Engineering, Hohai University, Nanjing 210098, China
}

Correspondence should be addressed to Wei Gao; wgaowh@163.com

Received 26 April 2016; Revised 29 June 2016; Accepted 30 June 2016

Academic Editor: Hossein Moayedi

Copyright (C) 2016 Wei Gao et al. This is an open access article distributed under the Creative Commons Attribution License, which permits unrestricted use, distribution, and reproduction in any medium, provided the original work is properly cited.

To solve the main shortcoming of numerical method for analysis of the stability of rock slope, such as the selection the convergence condition for the strength reduction method, one method based on the minimum energy dissipation rate is proposed. In the new method, the basic principle of fractured rock slope failure, that is, the process of the propagation and coalescence for cracks in rock slope, is considered. Through analysis of one mining rock slope in western China, this new method is verified and compared with the generally used strength reduction method. The results show that the new method based on the minimum energy dissipation rate can be used to analyze the stability of the fractured rock slope and its result is very good. Moreover, the new method can obtain less safety factor for the rock slope than those by other methods. Therefore, the new method based on the minimum energy dissipation rate is a good method to analyze the stability of the fractured rock slope and should be superior to other generally used methods.

\section{Introduction}

The rock slopes can be found in many engineering applications, such as highways and constructions in mountainous area, open pit mines, and hydropower projects. It is very important to maintain the stability of those rock slopes for the engineering safety. Nowadays, there are two main methods for analysis of the stability of rock slope, which are theoretical method and numerical method [1]. As the main ones of the theoretical method, the limit equilibrium-based methods remain the most popular option in rock slope engineering. These conventional methods, however, are limited to simple slope geometries, basic loading condition and rigid body assumption. Practical rock slope stability problems are intricate in the aspects of topological geometry, material anisotropy, nonlinear behavior, in situ stress, and the presence of several coupled processes. Therefore, numerical method helps to forward the approximate solutions, which would have never been possible using the conventional theoretical methods. However, the strength reduction method is the main one of the numerical method. Nowadays, there are many studies using the strength reduction method for analysis of the stability of rock slope.
For example, the loosened block rock slope of Jinyang Grand Buddha in Taiyuan of China is analyzed by the strength reduction method using Fast Lagrangian Analysis of Continua (FLAC) software [2]. Using FLAC software, Jiang [3] analyzed the stability of the bedding rock slope by strength reduction method with the ubiquitous-joint criterion. Moreover, the relationships of the bedding plane inclination angle and the safety factor are discussed. To analyze the jointed rock slopes, the strength reduction method based on the discrete element method is proposed [4]. Moreover, the sensitivity analyses are performed to better understand the critical mechanisms that lead to slope failure and to discriminate between the respective roles played by intact rock and planes of weakness at the onset of failure. To contrast techniques and highlight their advantages and drawbacks, Alejano et al. [1] analyze six theoretical examples using a limit equilibrium method and a numerical method, Universal Distinct Element Code (UDEC), combined with the strength reduction method. The first five examples concern fully joint-controlled rock slopes, for which only rigid blocks are needed, whereas the sixth example refers to a partially joint-controlled rock slope. To deal with the stability of highway cut in Eastern Slovakia, the strength reduction technique based on finite 
element analysis is used. In this study, the Mohr-Coulomb model and jointed rock model are all applied [5]. In the studies of Liu et al. [6], a new approach for determining the safety factor and the corresponding critical slip surface of a layered rock slope subjected to seismic excitations is presented, through a case study based on the combination of the strength reduction technique and distinct element method. Moreover, according to this proposed method, the seismic safety factor and the critical slip surface of the slope are estimated and compared with those obtained by the pseudostatic approach, combined with the limit equilibrium method. Chen et al. [7] present a comparative study of methods used for the analysis of the stability of rock slopes for hydropower projects. The comparison concerns the application of the limit equilibrium method, the block element method, and the finite element method on the stability analysis of two hydropower engineering projects in China. In those numerical studies, the strength reduction method is used. Nowadays, the linear MohrCoulomb criterion is generally used in the strength reduction method. But it is widely accepted that the shear strength of the rock mass is a nonlinear function of stress level. To analyze the stability of rock slope, one nonlinear Hoek-Brown shear strength reduction technique is proposed [8]. Moreover, the implementation of the nonlinear shear strength reduction method is described detailedly and a rock slope example is selected to verify this new method.

Although the strength reduction method is widely used, it has some shortcomings, such as the expensive computation and convergence issues due to the nonlinear iterative computations [9]. The selection of the convergence condition especially is a very hard work. To solve this problem, one method based on the minimum energy dissipation rate is proposed. At last, one rock slope example is used to verify the proposed method.

\section{Strength Reduction Method Based on Minimum Energy Dissipation Rate}

The strength reduction method was first proposed by Zienkiewicz et al. in 1975 [10]. Its definition of the safety factor for a slope is often considered to be the ratio of the actual shear strength to the lowest shear strength of a rock or soil material that is required to maintain the slope in equilibrium [11]. The strength reduction method usually assumes the Mohr-Coulomb strength for slope materials. In this method, the gravitational acceleration is assumed as one constant. And, the parameters of shear strength of a rock or soil material $(c$ and $\phi)$ are reduced by one reduction factor $F$. Thus, new parameters $\left(c^{\prime}\right.$ and $\left.\phi^{\prime}\right)$ can be obtained. The new parameters are reduced by another reduction factor again. Until the slope is in the limit equilibrium state, in other words, when the reduction factor increases a little, the slope will collapse. At this moment, the corresponding reduction factor is the safety factor of the slope. In other words, this method involves successive reductions (by some trial factors) in the shear strength of slope materials until failure occurs. The critical reduction factor at which collapse occurs may be regarded as the safety factor of the slope. The equations for this method are as follows [11]:

$$
\begin{aligned}
\tau^{\prime} & =\frac{\tau}{F}=\frac{c+\sigma \tan \phi}{F}=\frac{c}{F}+\frac{\sigma \tan \phi}{F}=c^{\prime}+\sigma \tan \phi^{\prime}, \\
c^{\prime} & =\frac{c}{F}, \\
\phi^{\prime} & =\arctan \left(\frac{1}{F} \tan \phi\right),
\end{aligned}
$$

where $\tau$ and $\tau^{\prime}$ are shear stress of slope materials before and after reduction, respectively; $c$ and $c^{\prime}$ are cohesion of slope materials before and after reduction, respectively; $\phi$ and $\phi^{\prime}$ are internal friction angle of slope materials before and after reduction, respectively; $\sigma$ is normal stress of slope materials; $F$ is the reduction factor.

A critical problem in the determination of the safety factor of a slope using the strength reduction method is the definition of the critical (or limit equilibrium) state. Nowadays, there are three main types of criteria for determining the critical state of the slope. The first one is the deformation characteristics of the slope, such as failure shear strain developed from the toe to the top of the slope [12]; the second one is stress distribution features, such as the connectivity of the plastic zone through the slope from the toe to the crest [13]; and the third one is the nonconvergence option of solutions in numerical modeling, which is widely taken as an indicator of the collapse of the slope [11]. However, different criteria for identifying the critical state of slope in numerical modeling may lead to significant differences in the results.

Generally, the critical criteria for FLAC software used in strength reduction method are the running steps and the maximum nodal unbalanced force [14]. Nevertheless, the maximum nodal unbalanced force is used widely. The nodal unbalanced force is the sum of forces acting on a node from its neighbouring elements. If a node is in equilibrium state, these forces should sum to zero. However, the maximum nodal unbalanced force should be given by the user. Thus, the safety factor determined by this method is affected by user's experience very largely. And the computing accuracy for the safety factor will be poor in most cases. To solve this problem, one method based on the minimum energy dissipation rate is proposed. In this method, the energy dissipation rate is applied to determine the status of the rock slope failure. The previous studies [15-17] show that rock slope failure is frequently controlled by a complex combination of discontinuities that facilitate kinematic release. The entire processes of the progressive slide surface development for rock slope related to crack initiation, propagation, coalescence, and degradation to eventual catastrophic failure are successfully captured. In other words, massive rock slope instability inherently requires the evolution of natural discontinuities through a gradual transition from a discontinuous-continuous to a fully discontinuous medium [17]. Therefore, to determine the status of the rock slope failure, it is very crucial to master the crack propagation in the rock slope. However, the energy dissipation is accompanied with the crack propagation $[18,19]$. Energy dissipation can describe the fracture process of rock mass very well $[19,20]$. Thus, using the energy dissipation 
rate, the safety factor of rock slope can be determined very accurately.

According to previous studies [19], the energy dissipation rate $\varphi(r, \theta, t)$ at any point nearby the crack tip whose coordinate is $(r, \theta)$ at any time $t$ during the fracture process of rock can be described as follows:

$$
\begin{aligned}
\varphi(r, \theta, t)=\sigma_{i j}(r, \theta) \dot{\varepsilon}_{i j}^{N}(r, \theta) \\
\quad=\frac{\dot{D}(t)}{[1-D(t)]^{2} E}\left\{\sigma_{x}^{2}(r, \theta)+\sigma_{y}^{2}(r, \theta)+\sigma_{z}^{2}(r, \theta)\right. \\
\quad-2 \mu\left[\sigma_{x}(r, \theta) \sigma_{y}(r, \theta)\right. \\
\left.\quad+\sigma_{y}(r, \theta) \sigma_{z}(r, \theta)+\sigma_{z}(r, \theta) \sigma_{x}(r, \theta)\right] \\
\left.\quad+2(1+\mu)\left[\tau_{x y}^{2}(r, \theta)+\tau_{y z}^{2}(r, \theta)+\tau_{z x}^{2}(r, \theta)\right]\right\},
\end{aligned}
$$

where $r, \theta$ are polar coordinates when the crack tip is taking as the coordinate origin, $t$ is the time parameter of the rock failure process, $\sigma_{i j}(r, \theta)$ is the name stress parameter near the crack tip, $\dot{\varepsilon}_{i j}^{N}(r, \theta)$ is the unrecoverable strain-rate of the point $(r, \theta)$ at time $t, E$ is the nominal elastic modulus, $\mu$ is nominal Poisson's ratio, $D(t)$ is the damage variable at time $t$, and the other parameters are the stresses of the point $(r, \theta)$.

Moreover, when $t=0, D(t)=D(0)=0$, and when $t=t_{r}$, $D(t)=D\left(t_{r}\right)=1$. In other words, when $t=t_{r}$, the energy consumption process of the point finishes, and then the point is completely damaged.

According to the principles of the fracture mechanics [21], the stress field nearby the crack tip is

$$
\begin{aligned}
& \sigma_{x}(r, \theta)=\frac{K_{\mathrm{I}}}{\sqrt{2 \pi r}} \cos \frac{\theta}{2}\left(1-\sin \frac{\theta}{2} \sin \frac{3 \theta}{2}\right) \\
& -\frac{K_{\mathrm{II}}}{\sqrt{2 \pi r}} \sin \frac{\theta}{2}\left(2+\cos \frac{\theta}{2} \cos \frac{3 \theta}{2}\right), \\
& \sigma_{y}(r, \theta)=\frac{K_{\mathrm{I}}}{\sqrt{2 \pi r}} \cos \frac{\theta}{2}\left(1+\sin \frac{\theta}{2} \sin \frac{3 \theta}{2}\right) \\
& +\frac{K_{\mathrm{II}}}{\sqrt{2 \pi r}} \sin \frac{\theta}{2} \cos \frac{\theta}{2} \cos \frac{3 \theta}{2}, \\
& \sigma_{z}(r, \theta)=\mu\left[\sigma_{x}(r, \theta)+\sigma_{y}(r, \theta)\right] \\
& =2 \mu \frac{K_{\mathrm{I}}}{\sqrt{2 \pi r}} \cos \frac{\theta}{2}-2 \mu \frac{K_{\mathrm{II}}}{\sqrt{2 \pi r}} \sin \frac{\theta}{2}, \\
& \tau_{x y}(r, \theta)=\frac{K_{\mathrm{I}}}{\sqrt{2 \pi r}} \cos \frac{\theta}{2} \sin \frac{\theta}{2} \cos \frac{3 \theta}{2} \\
& +\frac{K_{\mathrm{II}}}{\sqrt{2 \pi r}} \cos \frac{\theta}{2}\left(1-\sin \frac{\theta}{2} \sin \frac{3 \theta}{2}\right), \\
& \tau_{y z}(r, \theta)=+\frac{K_{\mathrm{III}}}{\sqrt{2 \pi r}} \cos \frac{\theta}{2} \text {, } \\
& \tau_{z x}(r, \theta)=-\frac{K_{\mathrm{III}}}{\sqrt{2 \pi r}} \sin \frac{\theta}{2},
\end{aligned}
$$

where $K_{\mathrm{I}}, K_{\mathrm{II}}$, and $K_{\mathrm{III}}$ are stress intensity factors for crack modes I, II and III, respectively.

Equation (3) is substituted into (2); the following equation can be obtained:

$$
\begin{aligned}
& \varphi(r, \theta, t)=\sigma_{i j}(r, \theta) \dot{\varepsilon}_{i j}^{N}(r, \theta)=\frac{1+\mu}{4 \pi r} \\
& \cdot \frac{\dot{D}(t)}{[1-D(t)]^{2} E}\left\{(3-4 \mu-\cos \theta)(1+\cos \theta) K_{\mathrm{I}}^{2}\right. \\
& +4 \sin \theta(2 \mu-1+\cos \theta) K_{\mathrm{I}} K_{\mathrm{II}} \\
& +[4(1-\mu)(1-\cos \theta)+(1+\cos \theta)(3 \cos \theta-1)] \\
& \left.\cdot K_{\mathrm{II}}^{2}+4 K_{\mathrm{III}}^{2}\right\} .
\end{aligned}
$$

According to the least energy consumption principle [22], for one point nearby crack tip, the crack propagation orientation must be the orientation along which the energy dissipation rate is minimum. Thus, the orientation angle of crack propagation must satisfy the following conditions:

$$
\begin{gathered}
\frac{\partial \varphi(r, \theta, t)}{\partial \theta}=0, \\
\frac{\partial^{2} \varphi(r, \theta, t)}{\partial \theta^{2}}>0 .
\end{gathered}
$$

Based on (4) and (5), the orientation angle of crack propagation $\theta_{\min }$ along which the energy dissipation rate is minimum can be obtained. The minimum energy dissipation rate $\left.\varphi_{c}(t)\right|_{t=0}$ is one material parameter which can be determined by the test of rock material fracture. Thus, there exist the following equation:

$$
\left.\varphi_{\min }(r, \theta, t)\right|_{t=0}=\left.\varphi\left(r, \theta_{\min }, t\right)\right|_{t=0}=\left.\varphi_{c}(t)\right|_{t=0} .
$$

According to (6), along the orientation whose angle is $\theta_{\min }$, when the energy dissipation rate at the point nearby crack tip reaches the critical value $\left.\varphi_{c}(t)\right|_{t=0}$, the crack propagation begins.

According to the conditions that $\theta_{\min }=0, K_{\mathrm{II}}=K_{\mathrm{III}}=0$, $t=0$, and $K_{\mathrm{I}}=K_{\mathrm{Ic}}$, the minimum energy dissipation rate is

$$
\begin{aligned}
\left.\varphi_{c}(t)\right|_{t=0} & =\left.\frac{\dot{D}(t)}{[1-D(t)]^{2}}\right|_{t=0} \frac{(1+\mu)(1-2 \mu) K_{\mathrm{I} c}^{2}}{\pi r} \\
& =\frac{\left.(1+\mu)(1-2 \mu) \dot{D}(t)\right|_{t=0} K_{\mathrm{I} c}^{2}}{\pi r},
\end{aligned}
$$

where $K_{\mathrm{Ic}}$ is the fracture toughness for crack mode I, which is a material constant.

According to the principles of coordinate transformation, (7) can be transformed as

$$
\varphi_{c}(t)=\frac{(1+\mu)(1-2 \mu) K_{\mathrm{I} c}^{2}}{\pi x / \sin \theta},
$$

where $x$ is the maximum horizontal displacement.

To compute the minimum energy dissipation rate of rock slope failure, the maximum horizontal displacement must be 
computed beforehand. Therefore, the maximum horizontal displacement for rock slope can be obtained by the following Fish function of FLAC software:

$$
\begin{aligned}
& \text { deffind_max_disp } \\
& \text { p_gp_gp_head } \\
& \text { maxdisp_value }=0.0 \\
& \text { maxdisp_gpid }=0 \\
& \text { loop while p_gp \# null } \\
& \operatorname{disp\_ gp}=\operatorname{sqrt}\left(g p \_x d i s p\left(p \_g p\right) \wedge 2\right) \\
& \text { if disp_gp > maxdisp_value } \\
& \text { maxdisp_value }=\text { disp_g}_{-} p \\
& \text { maxdisp_gpid }=g p_{-} i d\left(p_{-} g p\right) \\
& \text { end if } \\
& p_{-} g p=g p_{-} n e x t\left(p_{-} g p\right) \\
& \text { end loop } \\
& \text { end } \\
& \text { find_max_disp } \\
& \text { print maxdisp_value maxdisp_gpid }
\end{aligned}
$$

With the obtained maximum horizontal displacement and (8), the minimum energy dissipation rate can be obtained be the following Fish function of FLAC software:

$$
\begin{aligned}
& \text { def } Y \\
& p_{\_} z=\text { zone_head } \\
& d_{\_} \mu= \\
& d_{\_} \pi= \\
& d_{\_} K_{\mathrm{I} c}= \\
& \text { loop while } p_{\_} z \# \text { null } \\
& r=\text { disp_x } x \sin \theta \\
& Y=(1+\mu)(1+\mu) * K_{\mathrm{Ic}} \wedge 2 / \pi r \\
& \text { Endloop } \\
& \text { end }
\end{aligned}
$$

where $\mu, \pi$, and $K_{\mathrm{Ic}}$ are constants that can be determined from the practical engineering conditions.

Based on the definition of safety factor by the minimum energy dissipation rate, the safety factor can be determined as follows.

Firstly, the minimum energy dissipation rate of the crack propagation is computed by an initial value of the reduction factor. When the values of the reduction factor increase, the minimum energy dissipation rate will decrease. Until the minimum energy dissipation rate approaches zero, the corresponding value of the reduction factor will be the critical value of the safety factor. In other words, when the minimum energy dissipation rate approaches zero, the crack will penetrate and coalesce; thus, the rock slope collapses.

Therefore, in this method, the energy dissipation rate is used to determine the slope stability status. Because the energy dissipation rate can estimate the moment of the crack

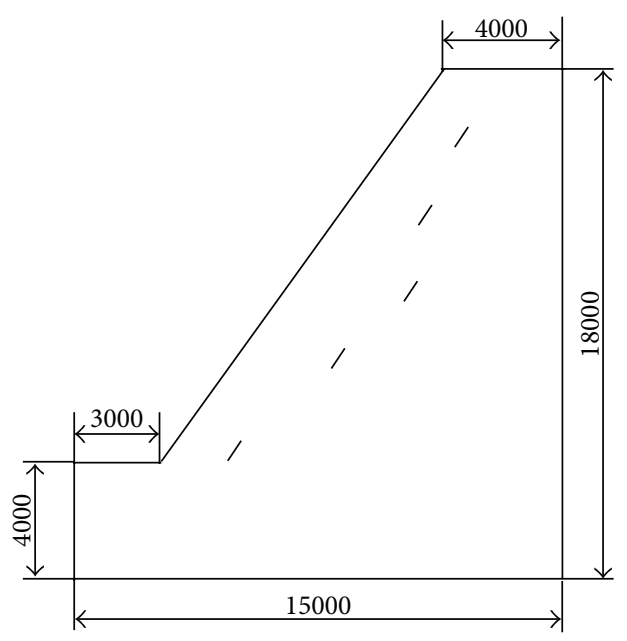

FIGURE 1: Dimensions of the mining rock slope.

propagation and coalescence for rock slope, the new method to determine the rock slope stability status based on the energy dissipation rate will be more precise than other methods.

\section{Engineering Example}

In this study, one mining rock slope in western China is used, whose width, height, and gradient are $15 \mathrm{~m}, 18 \mathrm{~m}$, and $60^{\circ}$, respectively. According to the geological condition, there exist five original cracks in the rock slope, whose lengths are all $100 \mathrm{~mm}$ and inclination is all $45^{\circ}$. The fracture toughness for all cracks is $K_{\mathrm{Ic}}=0.66 \mathrm{MPa} \cdot \mathrm{m}^{1 / 2}$. The dimension of the slope is shown in Figure 1, whose caption is dimensions of the mining rock slope.

The material parameters of the rock slope are as follows.

The elastic modulus $E$ is $26 \mathrm{GPa}$. The cohesion $c$ is $30 \mathrm{MPa}$. The internal friction angle $\phi$ is $30^{\circ}$. Poisson's ratio $\mu$ is 0.3 . The unit weight $\gamma$ is $2750 \mathrm{KN} / \mathrm{m}^{3}$. The uniaxial compressive strength is $18 \mathrm{MPa}$.

The numerical model of FLAC software for the rock slope is shown in Figure 2, whose caption is numerical model of rock slope.

3.1. Analysis by New Method. The main process to determine the safety factor of rock slope by this new method is as follows.

The Fish function programs are embedded into FLAC software. According to the strength reduction method, the values of $c$ and $\phi$ are reduced gradually. And, at the same time, the strength reduction factor of rock slope will increase gradually. When the strength reduction factor increases to one value, the computed minimum energy dissipation rate approaches zero. At this moment, the value of strength reduction factor will be the critical safety factor.

Because there are many original cracks in this rock slope, based on the engineering experience, the critical safety factor for this rock slope should not be very large. Therefore, in this study, the values of strength reduction factor are taken as 1.30, $1.35,1.40,1.45$, and 1.50. Using these values for strength 


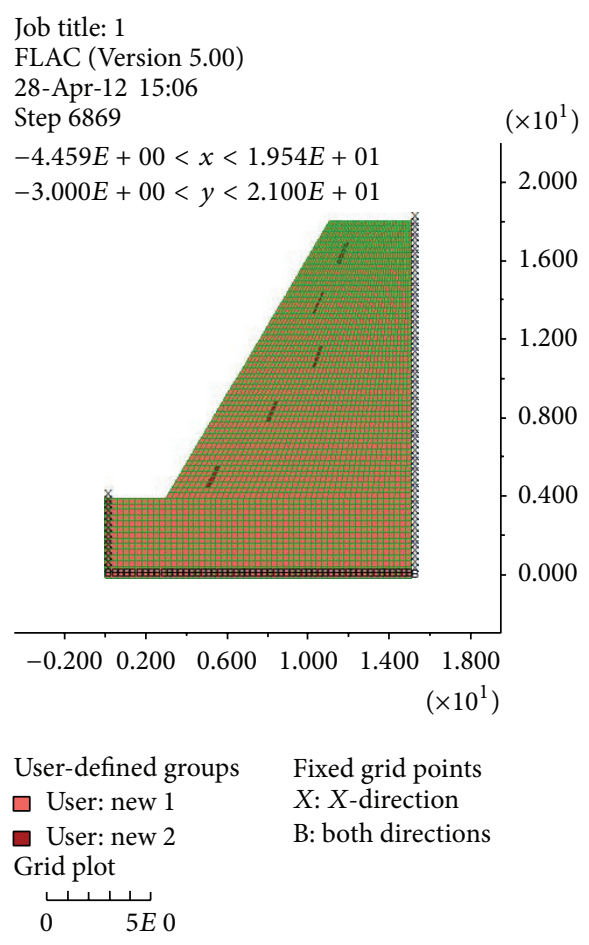

FIGURE 2: Numerical model of rock slope.

reduction factor, the corresponding maximum horizontal displacement and the minimum energy dissipation rate can be obtained as shown in Figures 3, 4, 5, 6, and 7, whose captions are results when strength reduction factor is $1.3,1.35$, $1.4,1.45$, and 1.5 , respectively. In these figures, the max- $x$ disp is the maximum horizontal displacement and MEDR is the minimum energy dissipation rate.

From the above figures, the following conclusions can be drawn.

When the strength reduction factor is 1.3 , at the beginning, the maximum horizontal displacement increases very quickly. And it can reach about $20 \mathrm{~cm}$ in 2 seconds. After about 4 seconds, the rock slope will arrive at stability status. At this moment, the maximum horizontal displacement will be $25 \mathrm{~cm}$. Moreover, at the beginning, the minimum energy dissipation rate is about 0.4 . As the time increases, the minimum energy dissipation rate increases too. And the final value of minimum energy dissipation rate is 1 . In other words, the rock slope is stable. Therefore, when the strength reduction factor is 1.3, the slope will not collapse; thus the safety factor should be larger than 1.3 .

When the strength reduction factor is 1.35 , at the beginning, the maximum horizontal displacement also increases very quickly. And it can reach about $50 \mathrm{~cm}$ in 1 second. After about 5 seconds, the rock slope will arrive at stability. At this moment, the maximum horizontal displacement will be $60 \mathrm{~cm}$. Moreover, at the beginning, the minimum energy dissipation rate is about 0.2 . As the time increases, the minimum energy dissipation rate increases too. And the final value of minimum energy dissipation rate is 1 too. In other words, the rock slope is stable. Therefore, when the strength reduction factor is 1.35 , the slope will not collapse too; thus the safety factor should be larger than 1.35.

When the strength reduction factor is 1.4 , although the final value of minimum energy dissipation rate also is 1 , the maximum horizontal displacement will arrive at a large value, which is $1.3 \mathrm{~m}$. In other words, although the slope is stable, its inner deformation is very large. Therefore, the rock slope should approach the instability.

When the strength reduction factor is 1.45 , the maximum horizontal displacement increases as an exponential law. Thus, the maximum horizontal displacement cannot be stable after 8 seconds. However, the minimum energy dissipation rate decreases to zero after 7 seconds. Therefore, at this moment, the cracks in the rock slope penetrate. And the slope fails.

On the other hand when the strength reduction factor increases to 1.5 , after only 4 seconds, the minimum energy dissipation rate will decrease to zero and the maximum horizontal displacement increases to larger than $3 \mathrm{~m}$. Therefore, the rock slope completely fails under this condition.

The damage status of the rock slope under the condition that the values of strength reduction factor are 1.3, 1.4, and 1.45 is shown in Figure 8, whose caption is damage state of rock slope. In FLAC software, the maximum shear strainrate is used to describe the damage state, shown by different colours in Figure 8. Moreover, in Figure 8, the arrows are the displacement vectors.

From Figure 8, it can be concluded that, when the strength reduction factor is 1.3 , the cracks in the rock slope are close, and the slope is stable. When the strength reduction factor increases to 1.4, the cracks in the rock slope open and propagate at some extent, especially, as for the cracks at the foot of the slope. However, at this moment, the cracks do not coalesce. While when the strength reduction factor is 1.45 , the cracks in the rock slope propagate very quickly and coalesce completely. Therefore, at this moment, the slope fails.

Therefore, the safety factor of this slope should be between 1.4 and 1.45. In this study, the middle value of 1.42 is selected as the safety factor of this slope.

3.2. Analysis by Generally Used Strength Reduction Method. To verify the new method proposed in this study and compare its results with those of other methods, the generally used strength reduction method is applied to analyze the rock slope.

Firstly, the initial force equilibrium status of the rock slope is computed. The curve of the unbalanced force for the rock slope system is shown in Figure 9, whose caption is curve of unbalanced force for the rock slope system. From Figure 9, at the beginning, the unbalanced force increases very quickly, and then it decreases gradually. Finally, it stabilizes to the value of zero. Therefore, the rock slope arrives to the status of the force equilibrium.

As for the status of force equilibrium, the horizontal stress and vertical stress in the rock slope are computed as shown in Figure 10, whose caption is stress distribution of the rock slope. In Figure 10, the stress is described by the different colours. In Figure 10(a), the horizontal stress increases from 


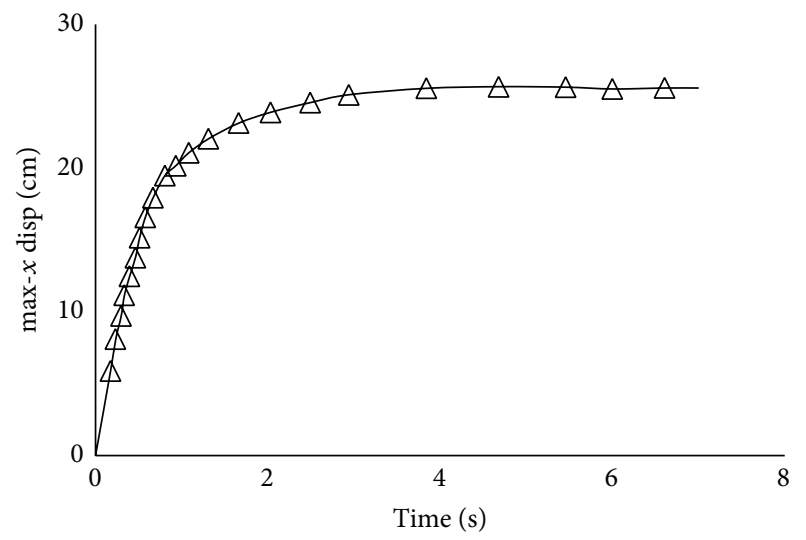

(a) Curve of maximum horizontal displacement

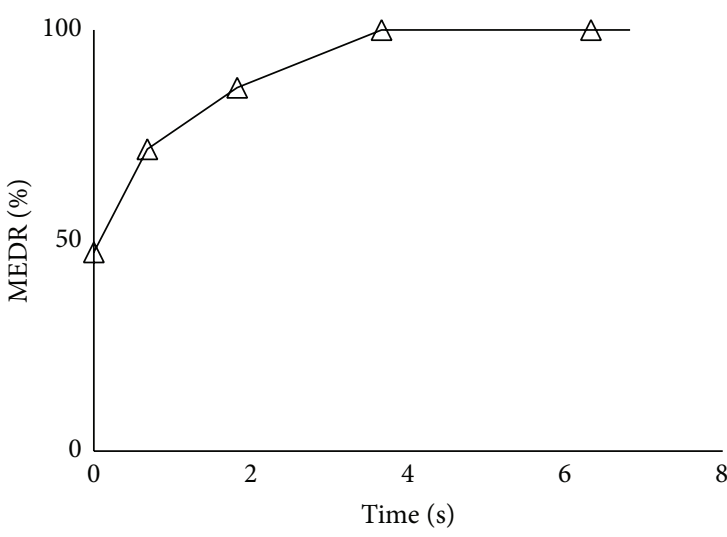

(b) Curve of minimum energy dissipation rate

FIgURE 3: Results when strength reduction factor is 1.3.

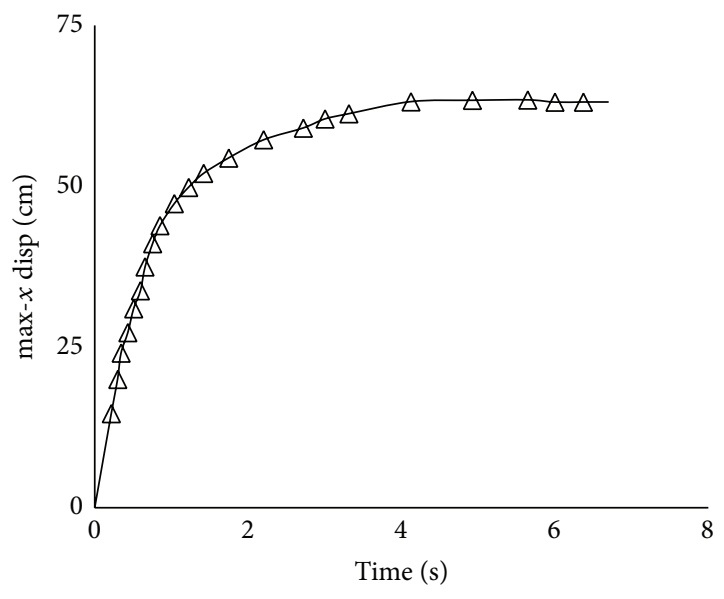

(a) Curve of maximum horizontal displacement

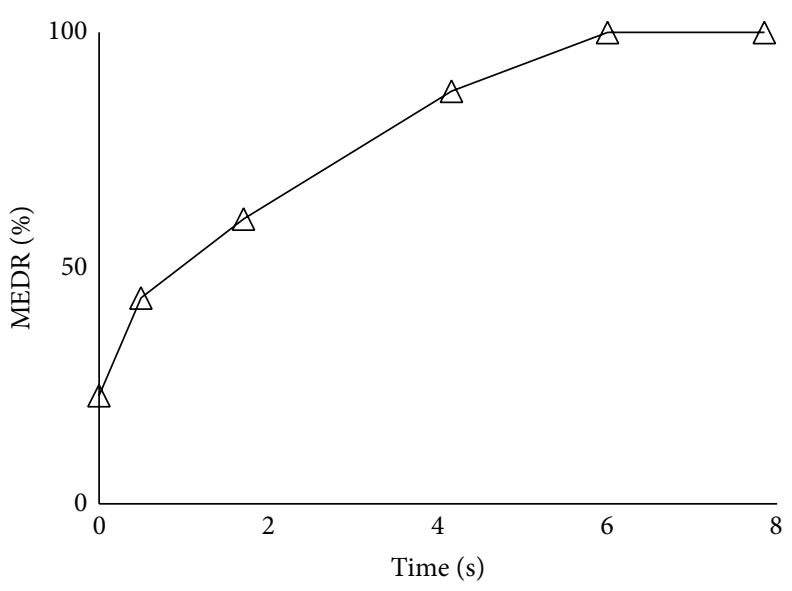

(b) Curve of minimum energy dissipation rate

FIGURE 4: Results when strength reduction factor is 1.35 .

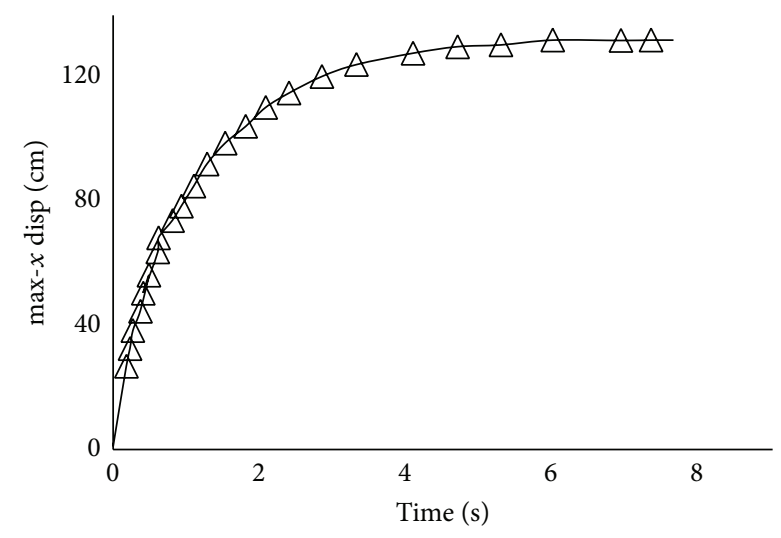

(a) Curve of maximum horizontal displacement

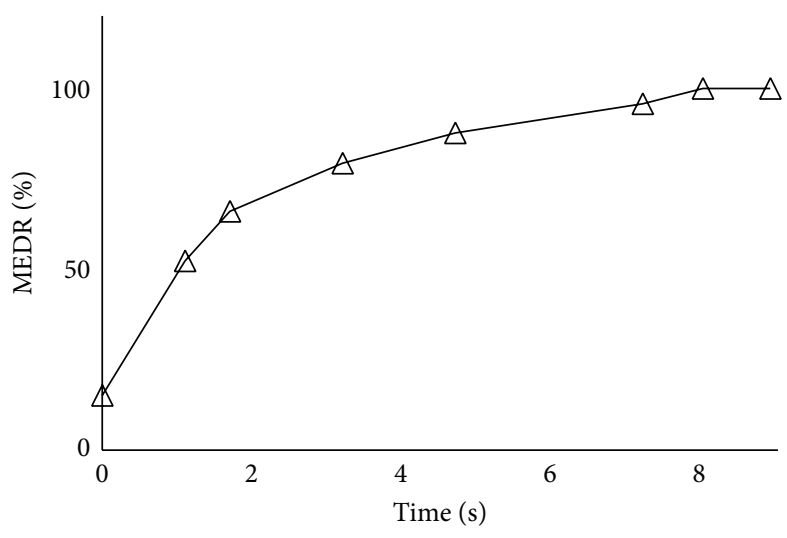

(b) Curve of minimum energy dissipation rate

FIGURE 5: Results when strength reduction factor is 1.4. 


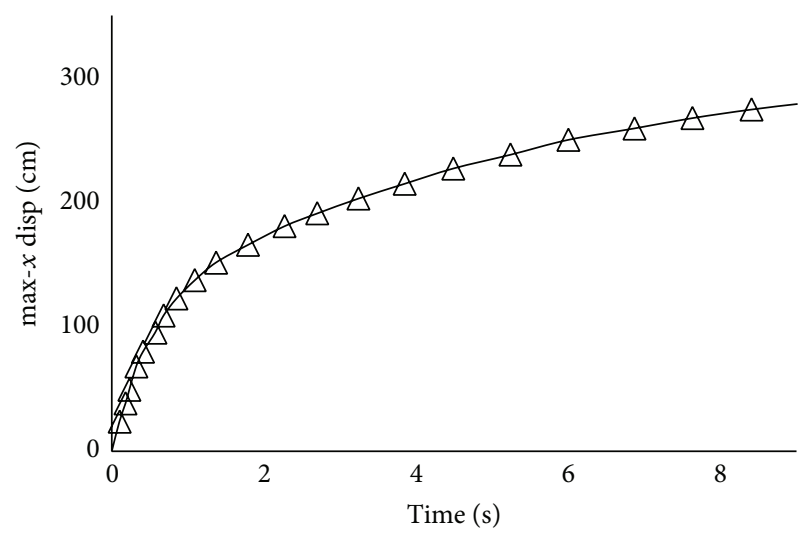

(a) Curve of maximum horizontal displacement

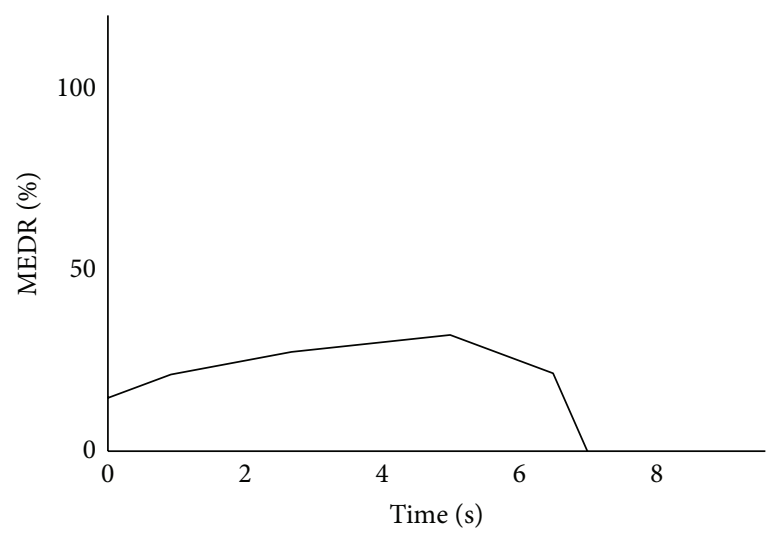

(b) Curve of minimum energy dissipation rate

FIGURE 6: Results when strength reduction factor is 1.45.

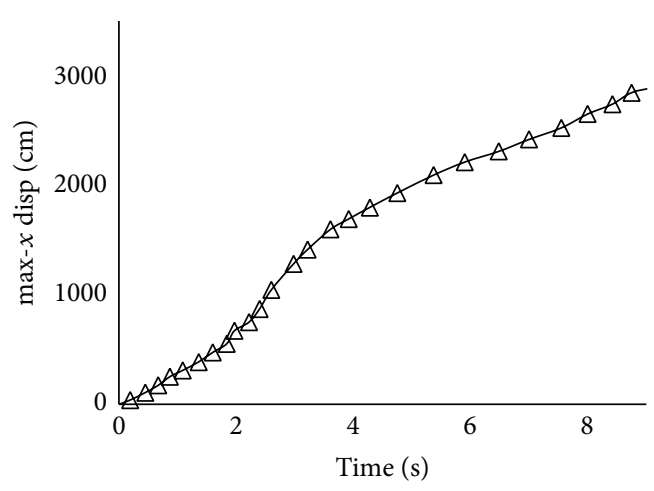

(a) Curve of maximum horizontal displacement

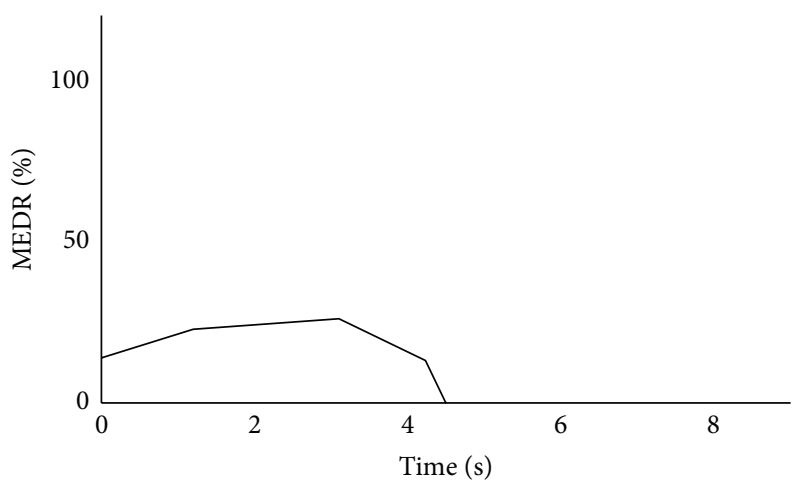

(b) Curve of minimum energy dissipation rate

FIGURE 7: Results when strength reduction factor is 1.5 .

left to right. And, in Figure 10(b), the vertical stress increases from top to floor.

From Figure 10, the stresses near the cracks are different from those in other zones. Moreover, all the horizontal stresses and vertical stresses at the tip of the cracks are obviously larger than those in other zones for the same area. Therefore, in the computing process by the strength reduction method, the stress will focus on the tip of cracks. Because the stress at the tip of cracks is larger than those in other zones, the damage will develop from the tip of cracks. And then the cracks will propagate and coalesce. At last, the rock slope will collapse. This phenomenon agrees with the progressive failure of rock slopes in previous studies $[15,17]$.

After the computation for the force equilibrium is complete, the safety factor of rock slope can be obtained by the strength reduction method. The computed safety factor is 1.51. The computed result is shown in Figure 11, whose caption is result of the generally used strength reduction method for the rock slope. In this figure, the maximum shear strain-rate, described as the failure state, is shown by different colours. And the arrows are the displacement vectors.

The computed safety factors by the new method is 1.42 and that by the generally used strength reduction method is
1.51. The difference of two values for the safety factors is not very large. Therefore, the new method based on the minimum energy dissipation rate is suitable to analyze the stability of the fractured rock slope. However, the safety factor by the new method is less than that by the generally used strength reduction method. In other words, the new method can obtain one more dangerous stability status than that by other methods. Therefore, the new method should be superior to other methods, such as generally used strength reduction method.

\section{Conclusions}

It is very important to analyze the stability of rock slope, which is a very complicated engineering problem. Nowadays, the numerical method, such as strength reduction method, is the main method to solve this problem. To overcome the shortcomings of the generally used strength reduction method, such as the expensive computation and convergence issues due to the nonlinear iterative computations, from the basic principle of rock slope failure, that is, the rock slope failure is the process of the propagation and coalescence for cracks in rock slope, one method based on the minimum energy dissipation rate is proposed. By analysis of one mining 


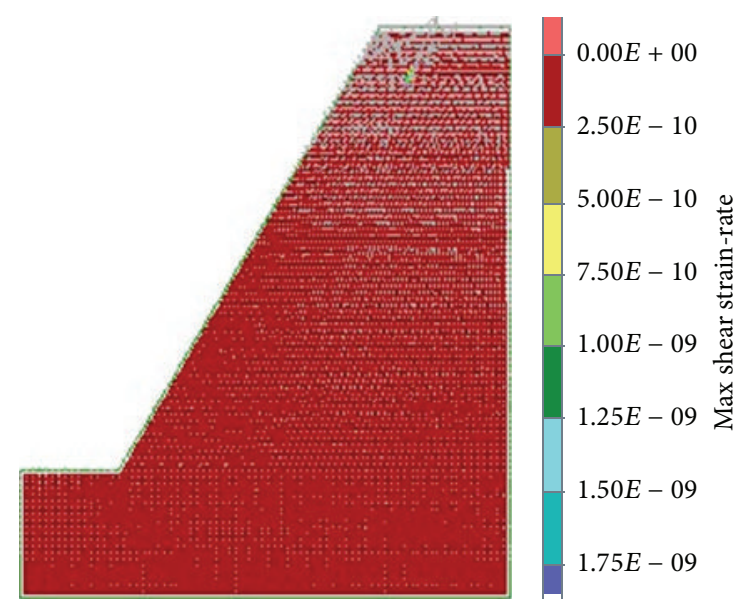

(a) Strength reduction factor of 1.3

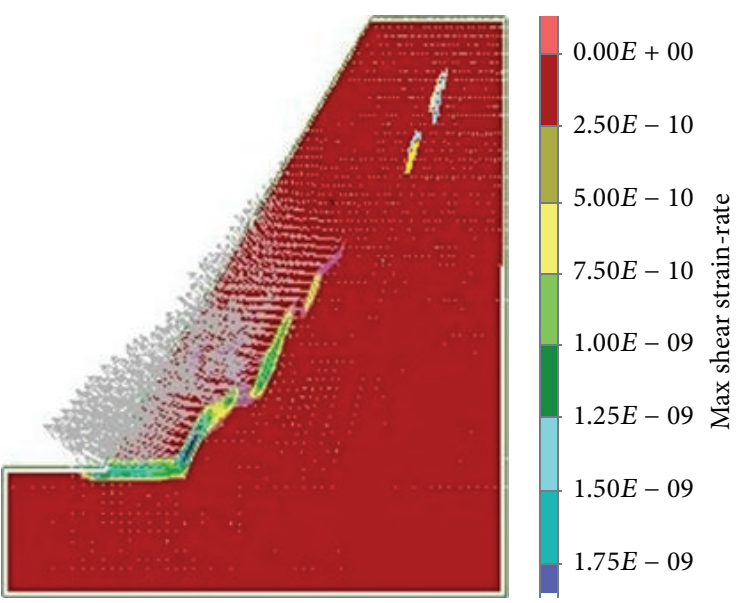

(b) Strength reduction factor of 1.4

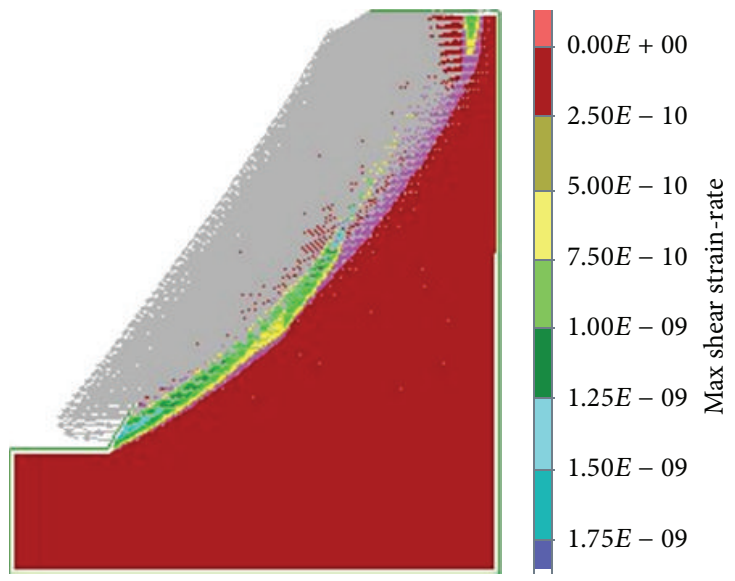

(c) Strength reduction factor of 1.45

FIgURE 8: Damage state of rock slope.

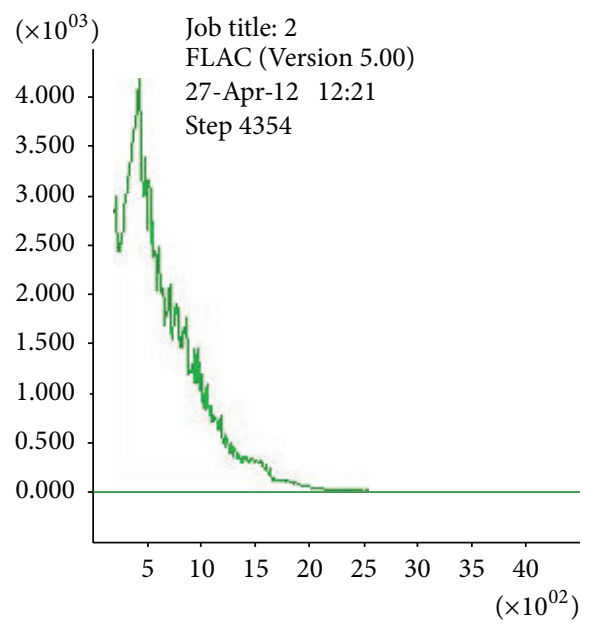

History plot $Y$-axis: 999 Max unbalanced force $X$-axis: number of steps

FIGURE 9: Curve of unbalanced force for the rock slope system. rock slope in western China, this new method is verified. From the studies, the following conclusions can be drawn:

(1) The massive rock slope instability inherently requires the evolution of natural discontinuities through a gradual transition from a discontinuous-continuous to a fully discontinuous medium. To determine the status of the rock slope failure, it is very crucial to master the crack propagation in the rock slope.

(2) The energy dissipation is accompanied with the crack propagation. Thus, energy dissipation can describe the fracture process of rock mass very well. Using the energy dissipation rate, the safety factor of rock slope can be determined very accurately.

(3) When the strength reduction factor is 1.4 , the cracks in the rock slope open and propagate at some extent. When the strength reduction factor increases to 1.45 , the cracks in the rock slope propagate very quickly and coalesce completely. Therefore, the safety factor of this slope should be between 1.4 and 1.45. 


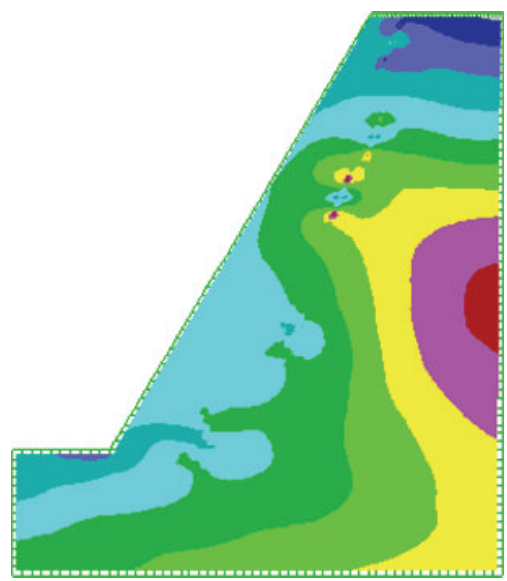

(a) Horizontal stress

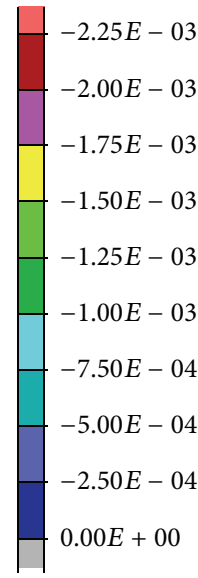

IGURE 10: Stress distribution of the rock slope.

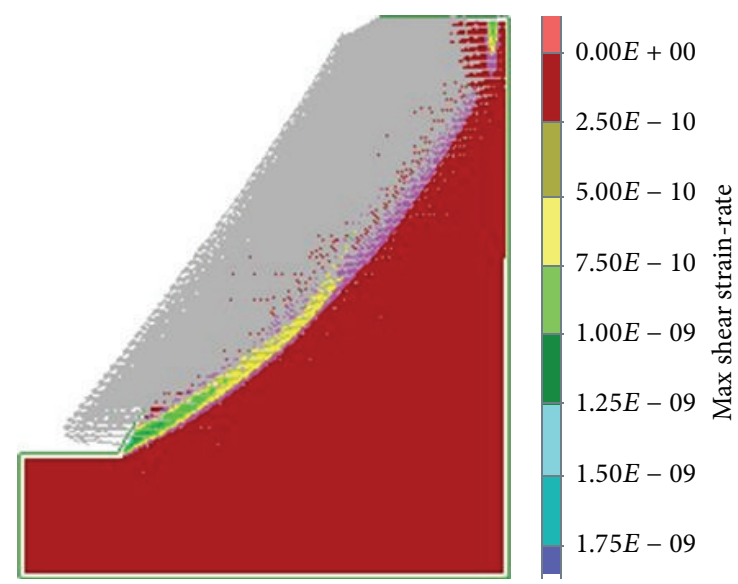

FIGURE 11: Result of the generally used strength reduction method for the rock slope.

(4) The computed safety factor by the new method is similar to that by the generally used strength reduction method. Therefore, the new method based on the minimum energy dissipation rate is a suitable method to analyze the stability of the fractured rock slope.

(5) The new method can obtain one more dangerous stability status of the fractured rock slope than that by generally used strength reduction method. Thus, the new method should be superior to the generally used strength reduction method.

Although the FLAC software is used in this study, using the same method, other numerical software programs, such as UDEC and Particle Flow Code (PFC), can all be used. Therefore, the new strength reduction method can be used widely by many numerical software programs.

\section{Competing Interests}

The authors declare that they have no competing interests.

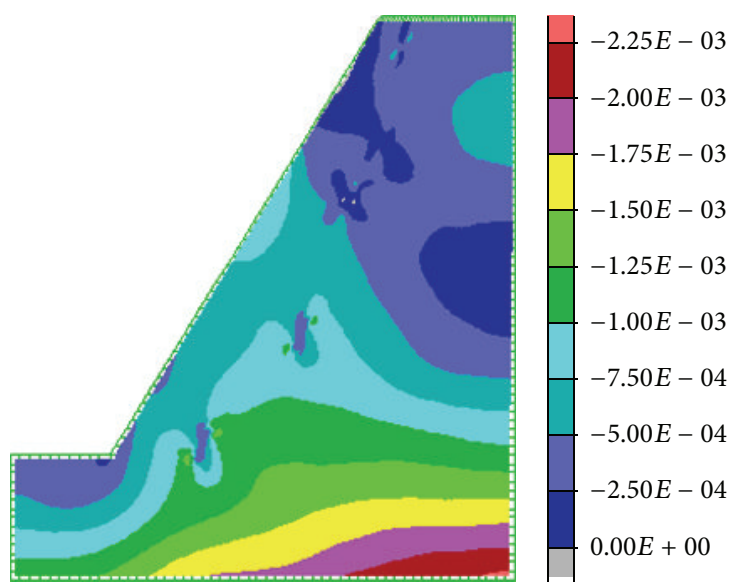

(b) Vertical stress

\section{Acknowledgments}

The financial supports from The Fundamental Research Funds for the Central Universities under Grant nos. 2014B17814, 2016B10214, 2014B07014, and B15020060 are all gratefully acknowledged.

\section{References}

[1] L. R. Alejano, A. M. Ferrero, P. Ramírez-Oyanguren, and M. I. Álvarez Fernández, "Comparison of limit-equilibrium, numerical and physical models of wall slope stability," International Journal of Rock Mechanics and Mining Sciences, vol. 48, no. 1, pp. 16-26, 2011.

[2] J. Z. Sun, X. F. Tian, X. D. Guan, Y. G. Yu, and X. S. Yang, "Stability analysis for loosened rock slope of jinyang grand buddha in Taiyuan, China," Earth Science Frontiers, vol. 15, no. 4, pp. 227-238, 2008.

[3] Q.-Q. Jiang, "Strength reduction method for slope based on a ubiquitous-joint criterion and its application," Mining Science and Technology, vol. 19, no. 4, pp. 452-456, 2009.

[4] L. Scholtès and F. V. Donzé, "A DEM analysis of step-path failure in jointed rock slopes," Comptes Rendus Mécanique, vol. 343, no. 2, pp. 155-165, 2015.

[5] J. Frankovská, M. Kopecký, J. Panuška, and J. Chalmovský, "Numerical modelling of slope instability," Procedia Earth and Planetary Science, vol. 15, pp. 309-314, 2015.

[6] Y. Q. Liu, H. B. Li, K. Q. Xiao, J. C. Li, X. Xia, and B. Liu, "Seismic stability analysis of a layered rock slope," Computers and Geotechnics, vol. 55, pp. 474-481, 2014.

[7] S.-H. Chen, W.-X. Qing, and I. Shahrour, "Comparative study of rock slope stability analysis methods for hydropower projects," Mechanics Research Communications, vol. 34, no. 1, pp. 63-68, 2007.

[8] W.X. Fu and Y. Liao, "Non-linear shear strength reduction technique in slope stability calculation," Computers and Geotechnics, vol. 37, no. 3, pp. 288-298, 2010.

[9] Y. M. Cheng, T. Lansivaara, and W. B. Wei, "Two-dimensional slope stability analysis by limit equilibrium and strength reduction methods," Computers and Geotechnics, vol. 34, no. 3, pp. 137-150, 2007. 
[10] O. C. Zienkiewicz, C. Humpheson, and R. W. Lewis, "Associated and non-associated visco-plasticity and plasticity in soil mechanics," Géotechnique, vol. 25, no. 4, pp. 671-689, 1975.

[11] E. M. Dawson, W. H. Roth, and A. Drescher, "Slope stability analysis by strength reduction," Géotechnique, vol. 49, no. 6, pp. 835-840, 1999.

[12] T. Matsui and K.-C. San, "Finite element slope stability analysis by shear strength reduction technique," Soils and Foundations, vol. 32, no. 1, pp. 59-70, 1992.

[13] H. Zheng, G. H. Sun, and D. F. Liu, "A practical procedure for searching critical slip surfaces of slopes based on the strength reduction technique," Computers and Geotechnics, vol. 36, no. 1-2, pp. 1-5, 2009.

[14] Itasca Consulting Group, FLAC-Fast Lagrangian Analysis of Continua, V5.0, Itasca Consulting Group, Minneapolis, Minn, USA, 2012.

[15] M.-A. Brideau, M. Yan, and D. Stead, "The role of tectonic damage and brittle rock fracture in the development of large rock slope failures," Geomorphology, vol. 103, no. 1, pp. 30-49, 2009.

[16] L. Scholtès and F.-V. Donzé, "Modelling progressive failure in fractured rock masses using a 3D discrete element method," International Journal of Rock Mechanics and Mining Sciences, vol. 52, pp. 18-30, 2012.

[17] L. N. Y. Wong and Z. Wu, "Application of the numerical manifold method to model progressive failure in rock slopes," Engineering Fracture Mechanics, vol. 119, pp. 1-20, 2014.

[18] S. Xiao, H.-L. Wang, B. Liu, and K.-C. Hwang, "The surfaceforming energy release rate based fracture criterion for elasticplastic crack propagation," Journal of the Mechanics and Physics of Solids, vol. 84, pp. 336-357, 2015.

[19] W. Gao, L. Wang, and S. He, "Study on rock fracture failure criterion based on energy principles," Advanced Science Letters, vol. 4, no. 3, pp. 869-874, 2011.

[20] W. Gao, L. Wang, and D. Yang, "Study on rock damage process based on least energy consumption principle," Advanced Science Letters, vol. 14, no. 1, pp. 380-383, 2012.

[21] T. L. Anderson, Fracture Mechanics: Fundamentals and Applications, CRC Press, Boca Raton, Fla, USA, 2005.

[22] Z. B. Zhou, Minimum Energy Principle and Its Applications, Science Press, Beijing, China, 2001 (Chinese). 

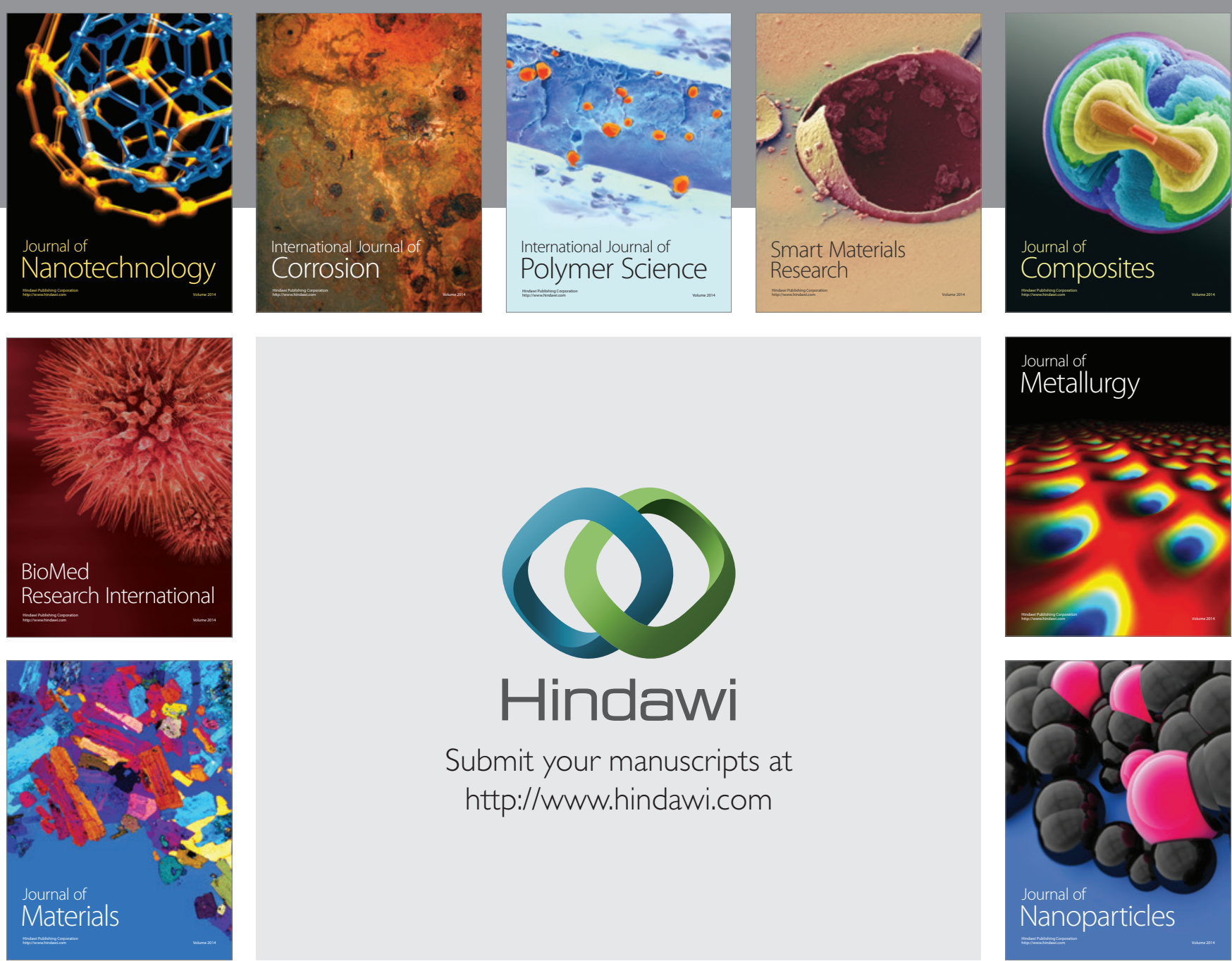

\section{Hindawi}

Submit your manuscripts at

http://www.hindawi.com

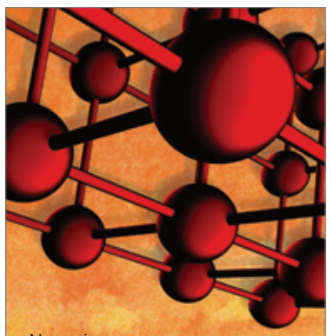

Materials Science and Engineering
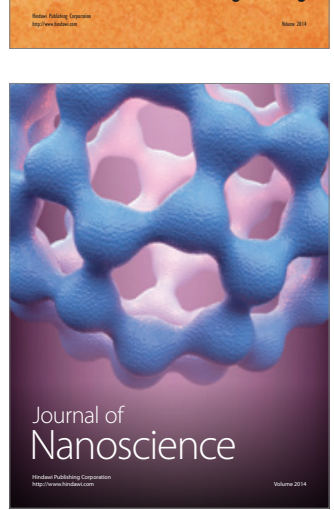
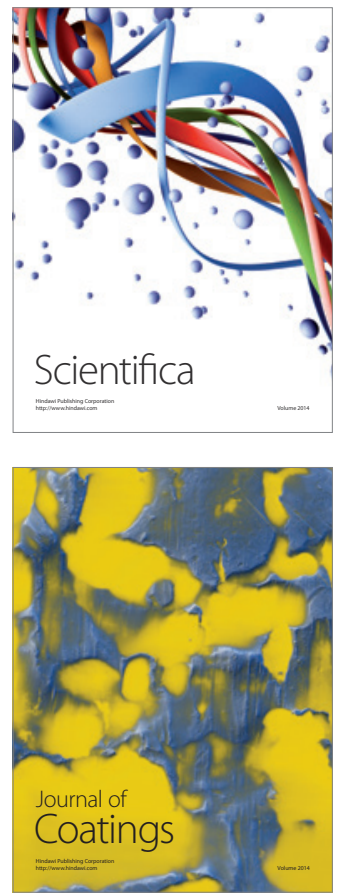
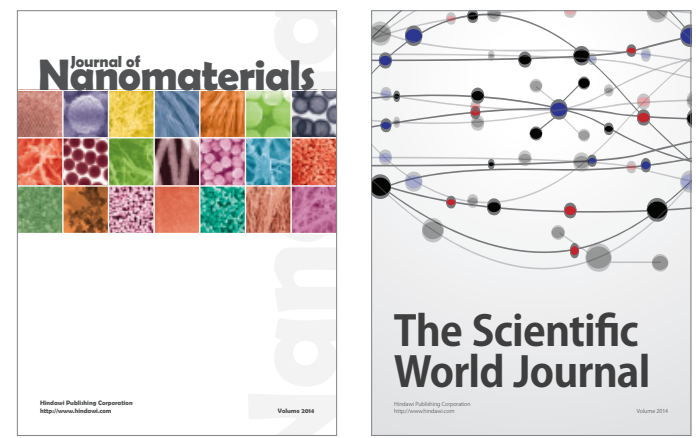

The Scientific World Journal
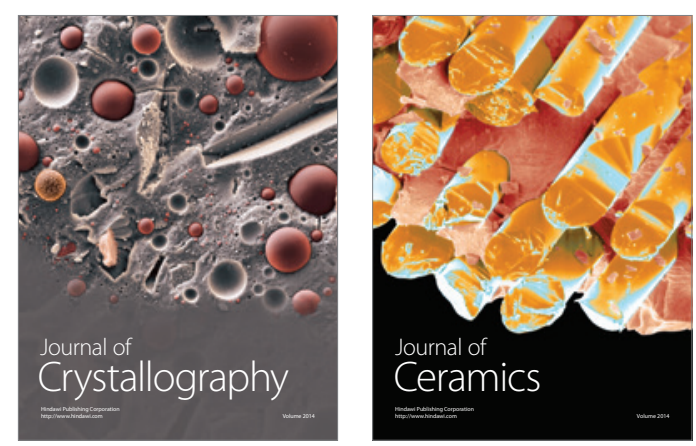
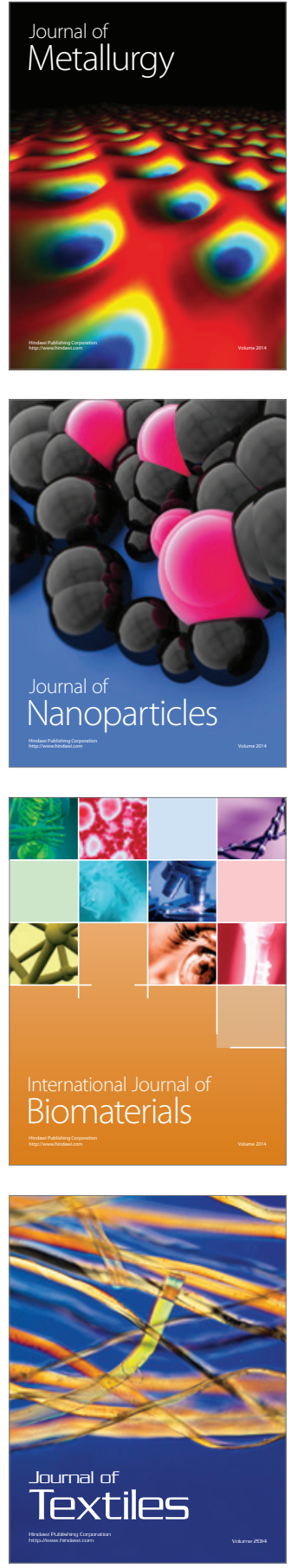